\title{
The CIREL Cohort: A Prospective Controlled Registry Studying the Real-Life Use of Irinotecan-Loaded Chemoembolisation in Colorectal Cancer Liver Metastases: Interim Analysis
}

\author{
Philippe L. Pereira ${ }^{1}$ Roberto Iezzi ${ }^{2} \cdot$ Riccardo Manfredi $^{2}$ - Francesca Carchesio ${ }^{2}$. \\ Zoltan Bánsághi $^{3} \cdot$ Elias Brountzos $^{4} \cdot$ Stavros Spiliopoulos $^{4} \cdot$ Javier J. Echevarria-Uraga $^{5}$. \\ Belarmino Gonçalves ${ }^{6}$ - Riccardo Inchingolo ${ }^{7} \cdot$ Michele Nardella $^{7}$. \\ Olivier Pellerin ${ }^{8} \cdot$ Maria Sousa $^{6}$ - Dirk Arnold ${ }^{9}$. Thierry de Baère ${ }^{10}$. \\ Fernando Gomez ${ }^{11,12}$ - Thomas Helmberger ${ }^{13} \cdot$ Geert Maleux $^{14}$ - Hans Prenen ${ }^{15}$. \\ Bruno Sangro $^{16} \cdot$ Bleranda Zeka $^{17} \cdot$ Nathalie Kaufmann $^{17} \cdot$ Julien Taieb $^{18}$
}

Received: 7 July 2020/ Accepted: 5 September 2020/Published online: 24 September 2020

(C) The Author(s) 2020

\begin{abstract}
Purpose Transarterial chemoembolisation (TACE) using irinotecan-eluting beads is an additional treatment option for colorectal cancer liver metastases (CRLM) patients that are not eligible for curative treatment approaches. This interim analysis focuses on feasibility of the planned statistical analysis regarding data distribution and
\end{abstract}

Electronic supplementary material The online version of this article (https://doi.org/10.1007/s00270-020-02646-8) contains supplementary material, which is available to authorised users.

Nathalie Kaufmann

kaufmann@cirse.org

1 Zentrum für Radiologie, Minimal-Invasive Therapien und Nuklearmedizin, SLK-Kliniken Heilbronn GmbH, Am Gesundbrunnen 20-26, 74078 Heilbronn, Germany

2 Dipartimento di Diagnostica per Immagini, Radioterapia Oncologica ed Ematologia, UOC di Radiologia Diagnostica ed Interventistica Generale, Fondazione Policlinico Universitario “A. Gemelli" IRCCS, Rome, Italy

3 Medical Imaging Center, Semmelweis University, Korányi Sándor u. 2, Budapest 1082, Hungary

4 Interventional Radiology Unit, 2nd Department of Radiology, School of Medicine, National and Kapodistrian University of Athens, Attikon University General Hospital, Rimini 1, Chaidari, 12462 Athens, Greece

5 Department of Radiology, Osakidetza Basque Health Service, Galdakao-Usansolo Hospital, Barrio Labeaga s/n, 48960 Galdakao, Spain

6 Department of Interventional Radiology, Portuguese Oncology Institute, Rua Dr. António Bernardino de Almeida, 4200-072 Porto, Portugal completeness, treatment intention, safety and health-related quality of life (HRQOL) of the first 50 patients prospectively enrolled in the CIrse REgistry for LifePearl ${ }^{\mathrm{TM}}$ microspheres (CIREL), an observational multicentre study conducted across Europe.

Methods In total, 50 patients $\geq 18$ years diagnosed with CRLM and decided to be treated with irinotecan-eluting LifePearl $^{\mathrm{TM}}$ microspheres TACE (LP-irinotecan TACE) by a multidisciplinary tumour board. There were no further inclusion or exclusion criteria. The primary endpoint is the categorisation of treatment intention, and secondary

7 Division of Interventional Radiology, Department of Radiology, Madonna delle Grazie Hospital, Via Montescaglioso, 75100 Matera, Italy

8 Assistance Publique Hôpitaux de Paris, Service de Radiologie Interventionnelle Vasculaire et Oncologique, Hôpital Européen Georges Pompidou, Université Paris Descartes, Sorbonne Paris-Cité, 20 Rue Leblanc, 75015 Paris, France

9 Asklepios Tumorzentrum Hamburg, AK Altona, Paul Ehrlich Str. 1, 22763 Hamburg, Germany

10 Service de Radiologie Interventionelle, Institut Gustave Roussy, 114 Rue Edouard Vaillant, 94800 Villejuif, France

11 Servicio de Radiodiagnóstico, Hospital Clínic de Barcelona, Calle Villarroel, 170, 08036 Barcelona, Spain

12 Department of Radiology, The Netherlands Cancer Institute, Plesmanlaan 121, 1066 CX Amsterdam, The Netherlands

13 Institut für Radiologie, München Klinik Bogenhausen Neuroradiologie und minimal-invasive Therapie, Englschalkinger Str. 77, 81925 Munich, Germany

14 Radiologie, UZ Leuven, Herestraat 49, 3000 Louvain, Belgium 
endpoints presented in this interim analysis are safety, treatment considerations and HRQOL.

Results LP-irinotecan TACE was conducted in $42 \%$ of patients as salvage therapy, $20 \%$ as an intensification treatment, $16 \%$ as a first-line treatment, $14 \%$ a consolidation treatment and $8 \%$ combination treatment with ablation with curative intent. Grade 3 and 4 adverse events were reported by $4 \%$ of patients during procedure and by $10 \%$ within 30 days. While $38 \%$ reported a worse, $62 \%$ reported a stable or better global health score, and $54 \%$ of patients with worse global health score were treated as salvage therapy patients.

Conclusion This interim analysis confirms in a prospective analysis the feasibility of the study, with an acceptable toxicity profile. More patients reported a stable or improved HRQOL than deterioration. Deterioration of HRQOL was seen especially in salvage therapy patients. Trial Registration NCT03086096.

Keywords TACE · Drug-eluting microspheres . Irinotecan · Chemoembolisation - Interim analysis

\section{Introduction}

Transarterial chemoembolisation (TACE) using irinotecaneluting beads is part of the loco-regional treatments toolbox to control colorectal cancer liver metastases (CRLM) in the $80 \%$ of patients that are not eligible for the only proven curative treatment options, surgery and thermal ablation $[1,2]$. Clinical trials and case-control studies have evaluated TACE using irinotecan in various settings and indications [3-13]. However, prospective real-world data, from which our understanding of how the treatment is used and how it can be improved, is still missing.

The CIrse REgistry for LifePearl ${ }^{\mathrm{TM}}$ microspheres (CIREL) was designed to prospectively capture data of CRLM patients decided to be treated with TACE using irinotecan-eluting LifePearl ${ }^{\mathrm{TM}}$ microspheres (LP-

15 Oncology Department, UZ Antwerp, Wilrijkstraat 10, 2650 Edegem, Belgium

16 Liver Unit, Clinica Universidad de Navarra-IDISNA and CIBEREHD, Av. de Pío XII 36, 31008 Pamplona, Spain

17 Clinical Research Department, Cardiovascular and Interventional Radiological Society of Europe, Neutorgasse 9, 1010 Vienna, Austria

18 Assistance Publique Hôpitaux de Paris, Service d'hepatogastroentérologie et d'oncologie digestive, Hôpital Européen Georges Pompidou, Université Paris Descartes, Sorbonne Paris-Cité, 20 Rue Leblanc, 75015 Paris, France irinotecan TACE). The study was carried out by interventional radiologists and supported by multidisciplinary teams, such as oncologists. This interim analysis was performed as a part of the CIREL study protocol in order to assess the feasibility of the study in terms the planned statistical analysis [14] and represents the first insight into the prospective real-life observation of the use of LPirinotecan TACE from 9 different European teams while also illustrating treatment considerations in terms of bead size and procedural medication and confirming the safety of TACE.

\section{Methods}

For a detailed description of CIREL methodology please refer to Pereira et al. [14].

\section{Study Design/Setting}

Data were supplied by 9 sites in 7 European countries by interventional radiologists who were invited to participate when having experience with the treatment and having performed at least one treatment with LP-irinotecan TACE and either a total of 40 treatments or at least 10 treatments in the last 12 months with any drug-eluting beads.

\section{Patients}

Eligible patients were $\geq 18$ years with histologically confirmed colorectal adenocarcinoma with liver-only or liverdominant metastases, and treatment with LP-irinotecan TACE (LifePearl ${ }^{\mathrm{TM}}$ microspheres, Terumo Europe N.V., Leuven, Belgium) decided in a multi-disciplinary tumour board. There were no further inclusion or exclusion criteria. Patients included in this 50-patient interim analysis were enrolled between February 2018 and June 2019. All aspects related to treatments and related to the follow-up protocol were performed at the treating physician's discretion, including whether treatments were performed under general or local anaesthesia, for which data were not collected.

\section{Study Objectives and Data Sources}

Patients and disease characteristics were collected through an e-CRF using OpenClinica 3. Automatic data checks and verifications were employed as far as possible, and additionally internal data logics were discussed via quarterly remote monitoring.

The primary outcome was the observation of usage of LP-irinotecan TACE by categorising treatment intention as: 
1. a first-line therapy for chemo-naïve or patients that have not received systemic chemotherapy after diagnosis of liver metastases,

2. a "consolidation" treatment with or without systemic chemotherapy for patients that have a tumour response or a stable disease on systemic chemotherapy,

3. an intensification treatment with concomitant therapy for patients with progressive disease that have received maximum 2 previous lines of treatment,

4. a salvage therapy for progressive disease patients that have received 3 or more previous lines of chemotherapy,

5. a combination treatment with ablation with curative intent

6. other if none of the previous categories apply.

For details regarding the grouping see supplementary Table 1.

The feasibility of the study was determined by the extent to which planned statistical analyses could be conducted when considering data completion and data distribution of baseline, safety and quality of life data.

Laboratory values were collected 1 to 9 days before LPirinotecan TACE treatments. Abnormal laboratory values during follow-ups were collected as adverse events. Bead size, the total dose infused, peri-procedural management and technical success (defined as complete delivery of the planned dose or complete stasis), as well as the number of procedures performed per lobe and additional medical and locoregional treatments, were registered and analysed.

All adverse events (AE) were classified according to CTCAE 4.03 (Cancer Institute's Common Terminology Criteria for Adverse Events 4.03 and 5.0; see supplementary Table 2) [15, 16] and were collected continuously. Health-related quality of life (HRQOL) questionnaires (QLQC30) were collected at 2 different timepoints: at baseline (1-9 days before treatment) and at the first followup (4-15 weeks after the last treatment session). HRQOL was assessed according to the EORTC QLQ-C30 Scoring Manual version 3.0 [17-19]. For the global health and the functional score, a high score indicates high health and for the symptom scale a low score indicates few symptoms. Cut-offs for clinically significant improvement $(+10$ for global health, functional score, -10 for symptom score) or deterioration ( -10 for global health, functional score, +10 for symptom score) compared to baseline were used [20].

\section{Bias}

Potential bias regarding data correctness and completeness was addressed by a quality assurance system including remote monitoring and data management. No source document verification was performed. Potential selection bias was addressed by contractually agreeing with all sites to present the possibility to participate to all potentially eligible patients. The number for non-inclusion was registered resulting in 56 potentially eligible patients having received LP-irinotecan TACE and 50 patients included in CIREL.

\section{Statistical Methods}

Baseline and treatment characteristics, as well as the primary endpoint of observed usage of LP-irinotecan TACE and secondary endpoints (laboratory data, adverse events and HRQOL), were evaluated using descriptive statistics. For continuous data, median (range) is shown. Categorical data are presented as counts (percentages). Data were plotted using RStudio under R3.6.1.

\section{Results}

\section{Patients and Disease Characteristics}

This interim report includes the prospectively captured real-life use of LP-irinotecan TACE as a treatment for CRLM in the first 50 patients enrolled. Median age was 66 years; 29 patients $(58 \%)$ were male. Patients and tumour characteristics are summarised in Table 1.

Liver metastases were synchronous in $68 \%$ and metachronous in $32 \%$ of patients. In total, $52 \%$ of those were located in the whole liver, 34\%/14\% limited to the right/ left lobe, respectively. In total, $34 \%$ had a proven RAS mutation, $48 \%$ RAS wild-type status (18\% unknown), $10 \%$ a proven BRAF mutation, 36\% wild-type status, 54\% unknown.

Before receiving LP-irinotecan TACE, 82\% (41 patients) had received prior systemic chemotherapy for metastatic disease. In total, $18 \%$ (9 patients) were pretreated with one line, $12 \%$ (6 patients) with two lines and $52 \%$ (26 patients) with 3 or more treatment lines. Of the overall population $36 \%$ received anti-angiogenic and $20 \%$ anti-EGFR targeted therapy. In 20\%, liver metastases had been resected with $4 \%$ of the total population having received adjuvant fluoropyrimidines and oxaliplatin and $4 \%$ having received adjuvant irinotecan. Prior ablation and intra-arterial liver-directed treatment were seen in $10 \%$ and $12 \%$ of patients.

\section{LP-Irinotecan TACE Treatment and Treatment Intention}

Information on 129 LP-irinotecan TACE treatment sessions in our 50 patients is shown in Table 2. 
Table 1 Tumour and patients' characteristics

\begin{tabular}{|c|c|c|c|c|c|}
\hline \multicolumn{6}{|l|}{ Primary tumour, $n(\%)$} \\
\hline Right-sided primary colon cancer (RSP) & \multicolumn{5}{|l|}{$10(20)$} \\
\hline Left-sided primary colon cancer (LSP) & \multicolumn{5}{|l|}{$29(58)$} \\
\hline Rectum cancer (LSP) & \multicolumn{5}{|l|}{$11(22)$} \\
\hline CEA increased & \multicolumn{5}{|l|}{$46(92)$} \\
\hline CA 19.9 increased & \multicolumn{5}{|l|}{$25(50)$} \\
\hline \multicolumn{6}{|l|}{ Primary tumour treatment, $n(\%)$} \\
\hline Surgery & \multicolumn{5}{|l|}{$44(88)$} \\
\hline Radiochemotherapy & \multicolumn{5}{|l|}{$6(12)$} \\
\hline Systemic chemotherapy & \multicolumn{5}{|l|}{$13(26)$} \\
\hline Targeted therapy & \multicolumn{5}{|l|}{$6(12)$} \\
\hline \multicolumn{6}{|c|}{ Eastern Cooperative Oncology Group (ECOG) performance status, $n(\%)$} \\
\hline 0 & \multicolumn{5}{|l|}{$36(72)$} \\
\hline 1 & \multicolumn{5}{|l|}{$11(22)$} \\
\hline 2 & \multicolumn{5}{|l|}{$3(6)$} \\
\hline \multicolumn{6}{|l|}{ Primary TNM status, $n(\%)$} \\
\hline Tis & 0 & No & $6(12)$ & M0 & $16(32)$ \\
\hline $\mathrm{T} 1$ & $5(10)$ & N1a & $11(22)$ & M1 & $28(56)$ \\
\hline $\mathrm{T} 2$ & $5(10)$ & N1b & $8(16)$ & Mx & $4(8)$ \\
\hline $\mathrm{T} 3$ & $28(56)$ & N1c & $3(6)$ & & \\
\hline \multirow[t]{3}{*}{$\mathrm{T} 4$} & \multirow[t]{3}{*}{$10(20)$} & $\mathrm{N} 2 \mathrm{a}$ & $5(10)$ & & \\
\hline & & $\mathrm{N} 2 \mathrm{~b}$ & $3(6)$ & & \\
\hline & & $\mathrm{Nx}$ & $12(24)$ & & \\
\hline
\end{tabular}

Molecular characterisation

$R A S, n(\%)$

Yes 17 (34)

No 24 (48)

N/A 9 (18)

$B R A F, n(\%)$

No 18 (36)

N/A $27(54)$

Extrahepatic metastases

Yes, $n(\%) \quad 16(32)$

No, $n(\%) \quad 34(68)$

Median number (larger than $10 \mathrm{~mm})(\min , \max ) \quad 2(1,6)$

Location, $n(\%)$

Lymph nodes $\quad 5$ (31)

Peritoneum $1(6)$

Lung $\quad 12(75)$

Bones $1(6)$

Liver metastases

Time since primary cancer diagnosis, $n(\%)$

Median time for liver metastases since primary tumour diagnosis, years (min, max) $\quad 2(0.3,9.7)$

Synchronous ( $<6$ months) 34 (68)

Metachronous (> 6 months) 16 (32)

Location, $n(\%)$

Whole liver $26(52)$

Left liver lobe only

Right liver lobe only 17 (34) 
Table 1 continued

\begin{tabular}{lc}
\hline Liver tumour burden, $n(\%)$ & \\
$<25 \%$ & $33(66)$ \\
$25-50 \%$ & $13(26)$ \\
$>50 \%$ & $4(8)$ \\
Median size of the two largest lesions, $m$ m (min, max) & \\
Lesion 1 & $54(10$, \\
$132) \times 43,5(10,127)$ & \\
Lesion 2 & $30(9,94)$ \\
& $\times 25(8$, \\
Number of lesions, $n(\%)$ & $87)$ \\
1 & \\
$2-3$ & $8(16)$ \\
$4-10$ & $16(32)$ \\
$>10$ & $15(30)$ \\
Previous treatments for liver metastases, $n(\%)$ & $11(22)$ \\
Systemic chemotherapy & \\
1 line & \\
2 lines & $41(82)$ \\
3 or more lines & $9(18)$ \\
Anti-angiogenic targeted therapy & $6(12)$ \\
Anti-EGFR targeted therapy & $26(52)$ \\
Surgery & $18(36)$ \\
Adjuvant fluoropyrimidine & $10(20)$ \\
Adjuvant oxaliplatin & $10(20)$ \\
Intra-arterial treatment & $2(4)$ \\
& $2(4)$ \\
& $2(4)$ \\
& $5(10)$ \\
& $6(12)$ \\
\hline
\end{tabular}

In total, $50 \%$ of patients received treatments in only one liver lobe (unilobar), with a median number of 2 sessions per patient ( $78 \%$ right lobe, $22 \%$ left lobe). The median number of treatments where both lobes were targeted alternatingly (bilobar) per patient was 2.6 sessions. The median intended dose per session was $100 \mathrm{mg}(80 \%$ of patients) using a bead size of $100 \mu \mathrm{m}(86 \%)$. Treatments were considered technically successful in $100 \%$ of cases, showing either a complete stasis in $36 \%$ or a complete delivery of dose in $64 \%$.

For most patients, intention of LP-irinotecan TACE was salvage therapy $(42 \% ; 21$ patients), meaning the patients had progressive disease and received three or more lines of chemotherapy before. LP- irinotecan TACE treatment was intended in $20 \%$ (10 patients) as an intensification treatment with concomitant systemic therapy for patients with progressive disease but maximum two previous lines of chemotherapy and 14\% (7 patients) as a consolidation treatment with or without systemic chemotherapy for patients with stable disease. Only $16 \%$ and $8 \%$ (8 and 4 patients, respectively) of treatments were classified as first line for chemo-naive patients or patients that have not received prior systemic chemotherapy for the liver metastases and combination treatment with ablation with curative intent, respectively. Treatment intentions were distributed evenly across high-enrolling sites (supplementary Table 3).

When analysing peri-procedural medications, we observed different treatment strategies in different sites as seen in Fig. 1. While opioids were used in almost all sessions, some patients received additional medication such as local anaesthesia, additional non-opioid pain- or anti-inflammatory medication, anti-histamine, antibiotics and antiemetics.

\section{Safety and Toxicity}

Abnormal laboratory values of grade 3 or 4 were observed 7 times in 4 patients before the first treatment and 11 times in 5 patients before subsequent LP-irinotecan TACE treatments (Table 3). Haematological, renal and hepatic toxicity was only observed by single abnormal laboratory 
Table 2 Treatment intentions for LP-irinotecan TACE and treatments' characteristics

\begin{tabular}{|c|c|}
\hline \multicolumn{2}{|l|}{ Treatment intention $n(\%)$} \\
\hline First-line treatment & $8(16)$ \\
\hline Consolidation or closing treatment with or without systemic therapy & $7(14)$ \\
\hline Intensification of treatment with concomitant systemic therapy & $10(20)$ \\
\hline $\begin{array}{l}\text { Rescue salvage treatment/end-stage treatment in progressive patients pretreated with systemic therapy, with or without } \\
\text { concomitant systemic therapy }\end{array}$ & $21(42)$ \\
\hline Combination treatment with ablation with a curative intent & $4(8)$ \\
\hline Unilobar treatment $n(\%)$ & $25(50)$ \\
\hline Median number of sessions (min, max) & $2(1,4)$ \\
\hline Right lobe & 39 \\
\hline Left lobe & 13 \\
\hline Bilobar treatment $n(\%)$ & $25(50)$ \\
\hline Right lobe & 45 \\
\hline Left lobe & 32 \\
\hline Median number of sessions per patient (min, max) & $2,6(1,5)$ \\
\hline \multicolumn{2}{|l|}{ LP-irinotecan TACE treatments $(n=129)$} \\
\hline Median intended dose per session, mg (min, max) & $\begin{array}{c}100(25, \\
100)\end{array}$ \\
\hline \multicolumn{2}{|l|}{ Bead size, $n(\%)$} \\
\hline $100 \pm 25 \mu \mathrm{m}$ & $111(86)$ \\
\hline $200 \pm 50 \mu \mathrm{m}$ & $17(13)$ \\
\hline $400 \pm 50 \mu \mathrm{m}$ & $1(1)$ \\
\hline \multicolumn{2}{|l|}{ Treatment technically successful $n(\%)$} \\
\hline Yes & $129(100)$ \\
\hline \multicolumn{2}{|l|}{ Technical success related to $n(\%)$} \\
\hline Complete stasis & $45(36)$ \\
\hline Complete delivery of the dose & $82(64)$ \\
\hline
\end{tabular}

values of grade 3 and 4 in individual patients with no increase compared to abnormal laboratory values of grade 3 and 4 before the first treatment session.

Adverse events (AE) are summarised in Table 4. In total, 33 peri-interventional AEs were reported, with $26 \%$ of patients having experienced at least one AE. Most notably, patients were experiencing grade 1 and grade 2 post-embolisation syndrome, which is defined as pain, fever or nausea/vomiting, with grade 1 pain being reported most frequently.

Within 30 days following the last treatment, 20\% (10 patients) experienced at least one AE. Grade 3 and 4 AEs were reported for $10 \%$ (5 patients). One patient experienced a grade $4 \mathrm{AE}$ (colonic obstruction and sepsis), as well as a grade $3 \mathrm{AE}$ (hepatic failure and blood bilirubin increase). Another patient experienced a grade $3 \mathrm{AE}$ (renal failure and hyperkalemia), and one patient a grade $3 \mathrm{AE}$ (infection and CPR increase) and one last patient experienced a grade $3 \mathrm{AE}$ (abscess). No adverse event resulting in mortality was reported in the 30 days after the last treatment.

\section{Health-Related Quality of Life Analysis}

Median HRQOL deteriorated over time, as both-median global health and median functional-scores decreased and the median symptom score increased (Fig. 2). Figure 2a, c, e shows the difference in median HRQOL for 34 patients for the first follow-up compared to the baseline scores of the same patients. Median global health, functional and symptom score before the first LP-irinotecan TACE treatment was 75.0, 91.1 and 8.3, respectively, and 66.7, 88.5 and 11.1, at the first follow-up. When looking at the patients individually, we see that for the majority of patients the global health, functional and symptom scores remained similar or improved (Fig. 2b, d, f).

In total, $62 \%$ of patients reported that the global health status stayed the same or improved $(18 \%)$ and $38 \%$ of patients showed a decrease of global health. For the functional score, $74 \%$ of patients reported a stable or increased $(3 \%)$ score and $26 \%$ of patients reported a worsening of the functional score. For the symptom score $82 \%$ reported stable or improved $(3 \%)$ score and $18 \%$ 

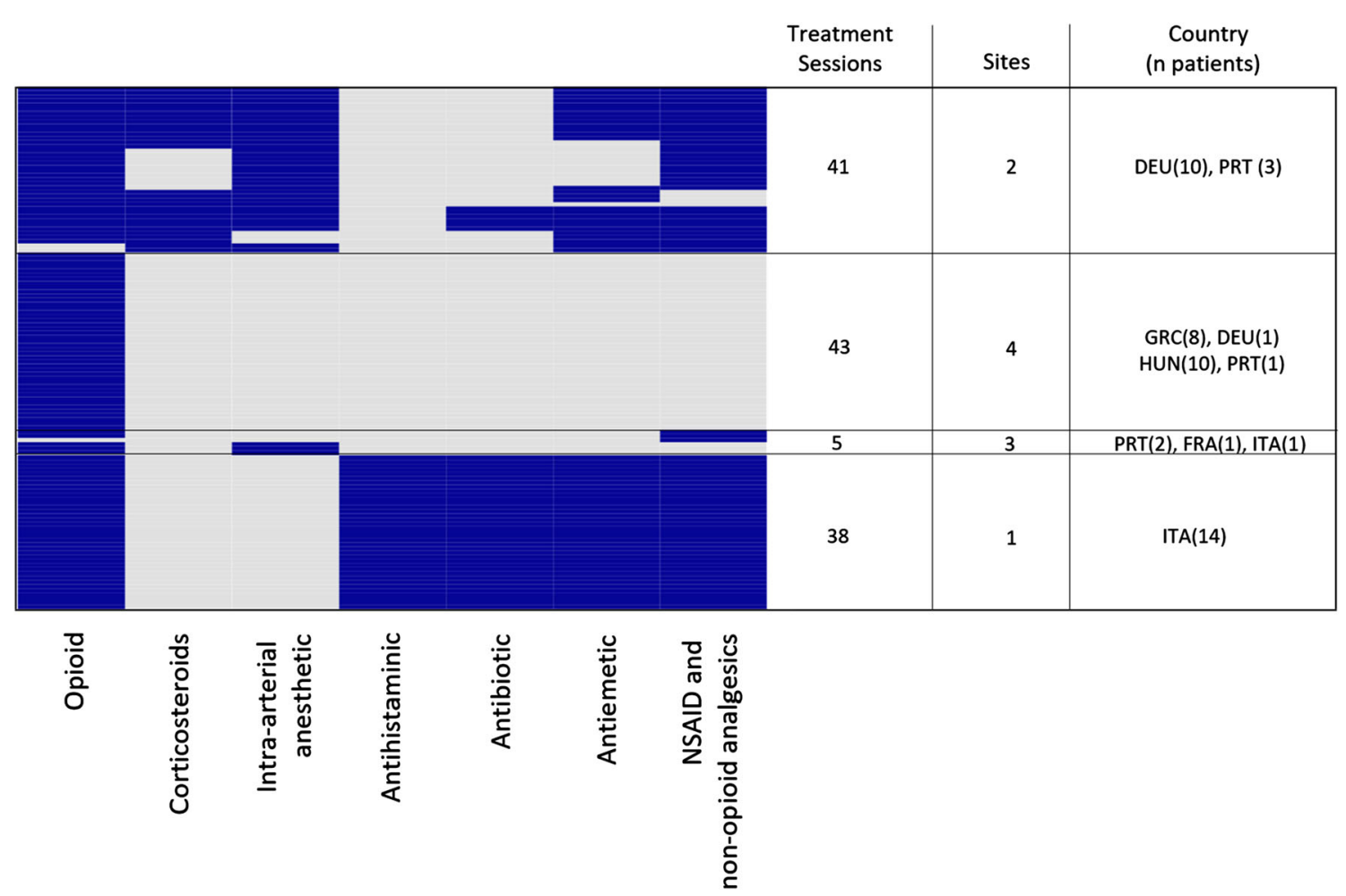

Fig. 1 Procedural medications used during LP-irinotecan TACE treatments. Depicts a heatmap of all medications used during each LP-irinotecan TACE treatment session $(n=127)$ divided into 4 groups based on similar medications used and a table listing the number of treatment sessions, different sites, as well as country (number of patients) per group. Each row represents one treatment, and each column represents the used class of medication. Coloured

reported worsening of the symptom score. When looking at patients with deterioration in global health, functionality and symptoms, we observed that $54 \%, 77 \%$ and $50 \%$, respectively, were salvage therapy patients (see Fig. 2b, d, f, red bars).

\section{Discussion}

While TACE is a first-line treatment option in intermediate hepatocellular carcinoma (HCC) [21, 22], CRLM TACE with irinotecan is a niche treatment with many competing interventional treatment alternatives such as thermal ablation, intra-arterial chemotherapy [23] and radioembolisation [1]. Additionally, systemic treatments including chemotherapy and targeted therapies, usually anti-VEGF or anti-EGFR antibodies, are used throughout the entire cancer continuum of care [1] and are the subject of many currently running trials [24-26]. Due to this competition of treatments experienced in daily clinical practice and considering that interventional radiologists are not always part of multidisciplinary tumour boards, difficulties in centre and patient enrolment were encountered in CIREL. Due to fields indicate that during the treatment the respective medication was used. 1 patient (in 2 treatments) where epidural anaesthesia was used in the absence of any other procedural medication is not shown. The heatmap was generated using the $\mathrm{R}$ heatmap function with the default clustering algorithm. Dendrograms for the clustering algorithm are not shown

this lower than expected enrolment, the CIREL Steering Committee decided to end patient enrolment after 30 months instead of the initially planned 36 months and to increase the follow-up period to allow capturing of highquality data and estimating meaningful survival data [14].

Despite these obstacles, CIREL will be the largest multicentre, prospective observational study on the real-life use of TACE with irinotecan using LifePearl ${ }^{\mathrm{TM}}$ microspheres in Europe and the first prospective study to comprehensively categorise treatment intention for irinotecan TACE in CRLM.

For this, it was considered whether patients had received previous systemic therapy, how many lines thereof, and at which stage of the cancer continuum of care the treatment was performed (supplementary Table 1). An additional group was LP-irinotecan TACE as a combination treatment with ablation with a curative intent. Typically, TACE with irinotecan is mainly used as a salvage therapy, with no consistent definitions of the number of previous lines of chemotherapy across already published studies [27-30] . Furthermore, evaluation of treatment intention is lacking. With LP-irinotecan TACE being used in $42 \%$ of patients as a salvage therapy, CIREL has not only prospectively 
Table 3 Pathological laboratory values before and after LP-irinotecan TACE treatments

\begin{tabular}{|c|c|c|c|c|}
\hline CTCAE 4.03. grade & Grade 1 & Grade 2 & Grade 3 & Grade 4 \\
\hline \multicolumn{5}{|c|}{ Max 7 days before first LP-irinotecan TACE treatment } \\
\hline Serum creatinine increased & 2 & 0 & 0 & 2 \\
\hline Bilirubin increased & 5 & 2 & 0 & 0 \\
\hline SGPT increased & 12 & 2 & 0 & 0 \\
\hline SGOT increased & 15 & 1 & 0 & 0 \\
\hline Albumin decreased & 0 & 1 & 0 & 0 \\
\hline LDH increased & 20 & N/A & N/A & N/A \\
\hline Alkaline phosphatase increased & 20 & 4 & 2 & 0 \\
\hline Neutrophils decreased & 1 & 0 & 0 & 1 \\
\hline Platelets decreased & 8 & 0 & 0 & 0 \\
\hline Lymphocytes decreased & 7 & 4 & 1 & 1 \\
\hline \multicolumn{5}{|c|}{ 1-9 days before consecutive treatments } \\
\hline Serum creatinine increased & 4 & 0 & 0 & 0 \\
\hline Bilirubin increased & 6 & 3 & 0 & 0 \\
\hline SGPT increased & 26 & 5 & 1 & 0 \\
\hline SGOT increased & 28 & 2 & 0 & 0 \\
\hline Albumin decreased & 0 & 3 & 0 & 0 \\
\hline LDH increased & 27 & N/A & N/A & N/A \\
\hline Alkaline phosphatase increased & 44 & 10 & 5 & 0 \\
\hline Neutrophils decreased & 2 & 0 & 0 & 1 \\
\hline Platelets decreased & 5 & 0 & 0 & 0 \\
\hline Lymphocytes decreased & 21 & 5 & 2 & 2 \\
\hline
\end{tabular}

One session from one patient was removed because the date of blood sampling was out of time range (48 days) evaluated and precisely described usage of LP-irinotecan TACE as a salvage treatment, but also differentiated between other treatment intentions, e.g. usage as a consolidation or intensification therapy. In this context, the results of CIREL will also improve our understanding of LP-irinotecan TACE used in clinical settings which are less commonly studied such as in chemotherapy-naive patients, only reported on in a few studies $[10,31]$. With a bigger sample size and in relation to these categories, effectiveness outcomes like overall survival and (hepatic) progression-free survival will be reported in the final analysis.

The vast majority $(86 \%)$ of treatments was performed using $100-\mu \mathrm{m}$ beads. While available bead sizes range from small $(70-150 \mu \mathrm{m})$ to very large $(500-700 \mu \mathrm{m})$, smaller beads $(70-150$ or $100-300 \mu \mathrm{m})[32,33]$ are recommended based on studies, suggesting that best clinical results can be achieved with small beads [34-37]. However, whether $70-150-\mu \mathrm{m}$ beads lead to better results is still a matter of debate [38] and a recent study by Boeken et al. showed no significant difference regarding patient outcome when using different bead sizes [39].

In contrast to doxycycline-TACE in intermediate HCC, there is no standardisation of peri-procedural management for irinotecan TACE. As expected, this has resulted in vast differences in procedural medications being reported in CIREL, which has been already reported in the literature [40]. In response to the need for treatment standardisation, Iezzi et al. recently published recommendations, suggesting the continuous infusion of opioid and non-steroidal anti-inflammatory medication to reduce peri-procedural pain. At the physician's discretion they suggest the prophylactic administration of antibiotics before and after treatment as well as the intra-arterial lidocaine administration directly prior to bead injection [33].

The appropriate peri-procedural management is important to control treatment-associated adverse events such as the commonly experienced grade 1 or 2 post-embolisation syndrome (PES). PES (grade 1) is also the most common adverse events (AE) by far in this interim analysis. While the number of reported grade 1 and grade 2 AEs varied highly, most studies reported none [36, 41, 42]] or below $10 \%[10,27,31,43]$ adverse events of grade 3 and 4 . This is in line with what was observed in CIREL with $10 \%$ of grade 3 reported adverse events and only $4 \%$ of grade 4 in this interim analysis. However, correlating different periprocedural medications and treatment strategies with adverse events is beyond the scope of this interim analysis. 
Table 4 Adverse events experienced peri-interventionally and within the first 30 days after LP-irinotecan TACE treatment

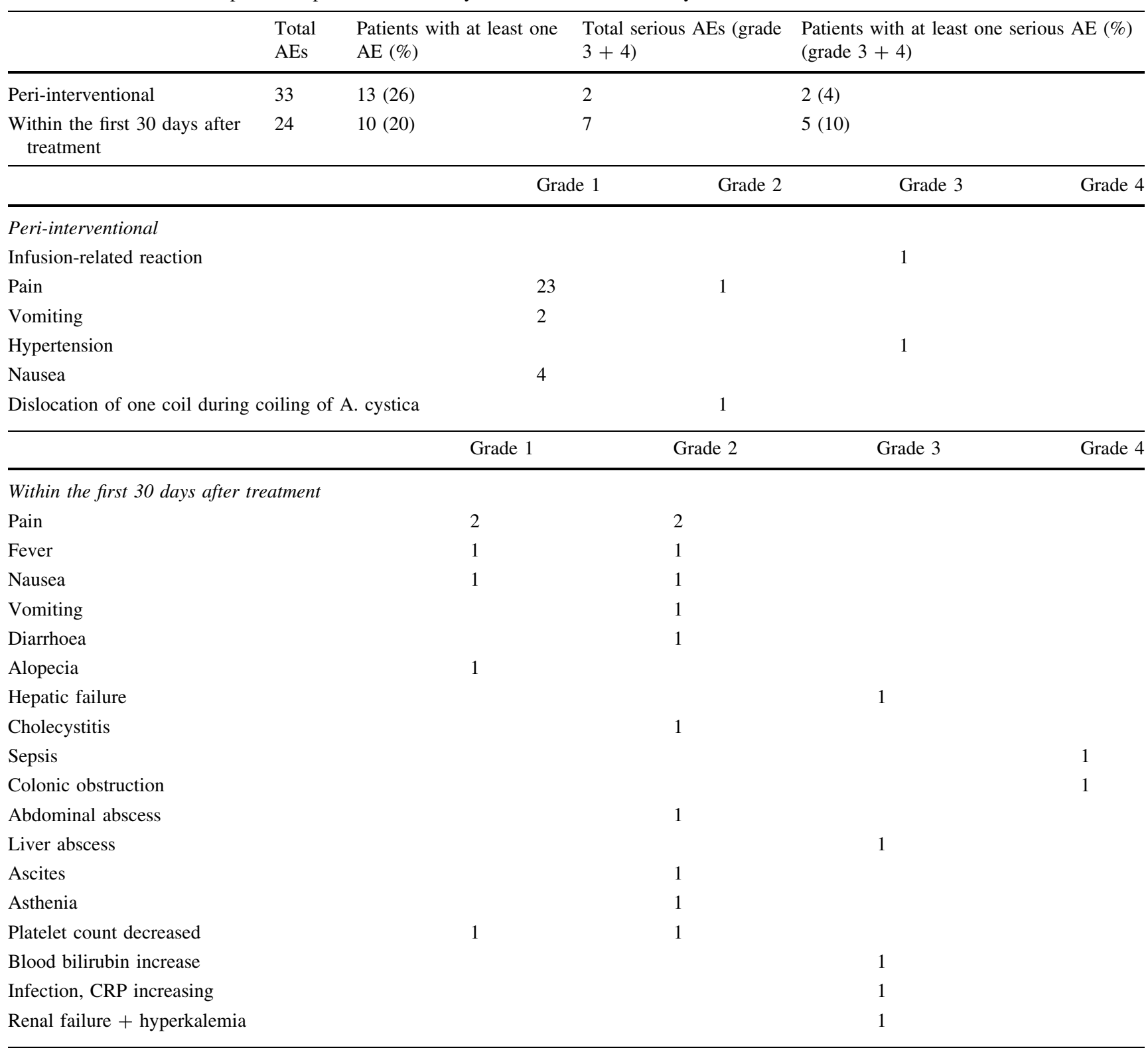

Another important aspect of palliative treatment is the maintenance of health-related quality of life (HRQOL), yet there are only few studies dedicated to studying HRQOL in TACE in CRLM [44-47]. Our data suggest improvement or maintenance of global health in $62 \%$, of functionality in $74 \%$ and of symptom score in $82 \%$ of patients. Deterioration in HRQOL global health score was observed especially for salvage therapy patients. While reporting maintenance or improvement of HRQOL, most studies have a limited number of participants [46, 47] or are not using patient-reported performance scales [45] and there is currently no comparable study describing the effect on irinotecan TACE on global health and functionality score. Previous findings using the Edmonton SAS questionnaire which correlates with the symptom score of the EORTC QLQ 30 in a comparable cohort to CIREL report improvement in $91 \%$ of patients [44] which is in line with our results regarding the symptom score. Using the comprehensive patient-reported EORTC QLQ 30-questionnaire scoring system, in the final analysis CIREL will give relevant insights into HRQOL for patients with different treatment intentions and into whether a gain in overall survival can be observed despite a deterioration of global health in salvage therapy patients. 
A

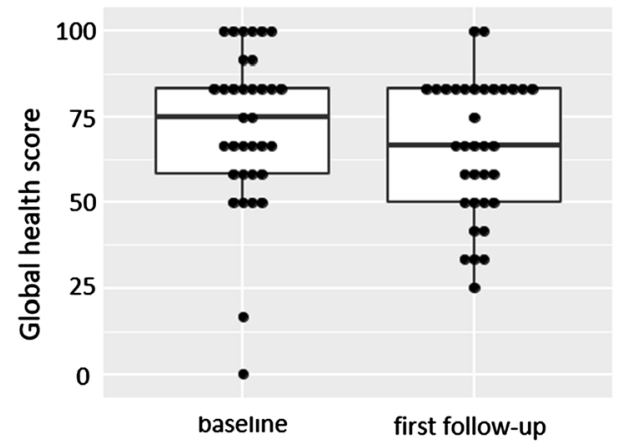

C

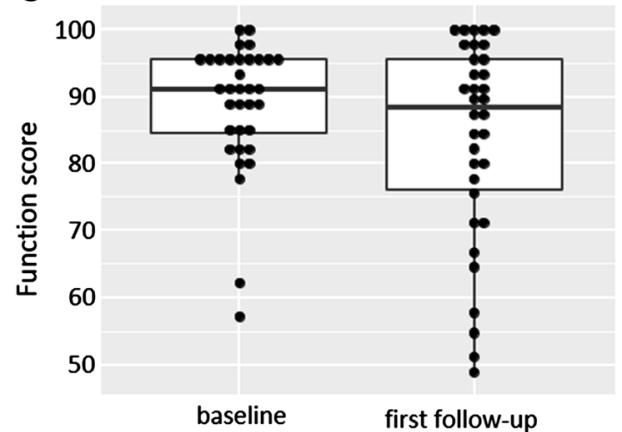

$\mathbf{E}$

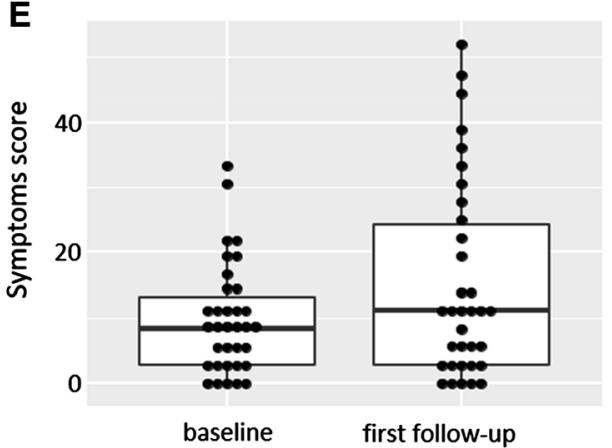

B

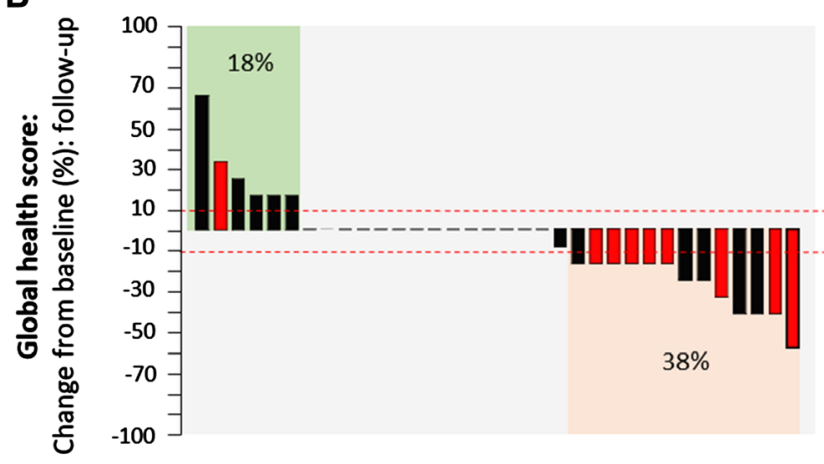

D

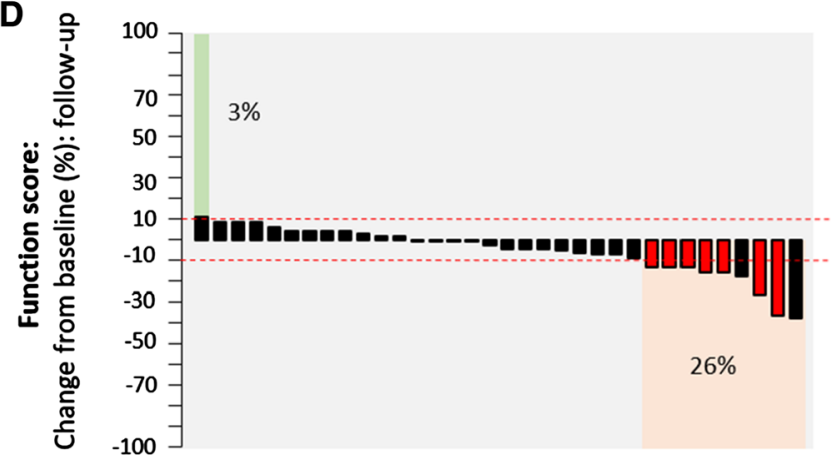

$\mathbf{F}$

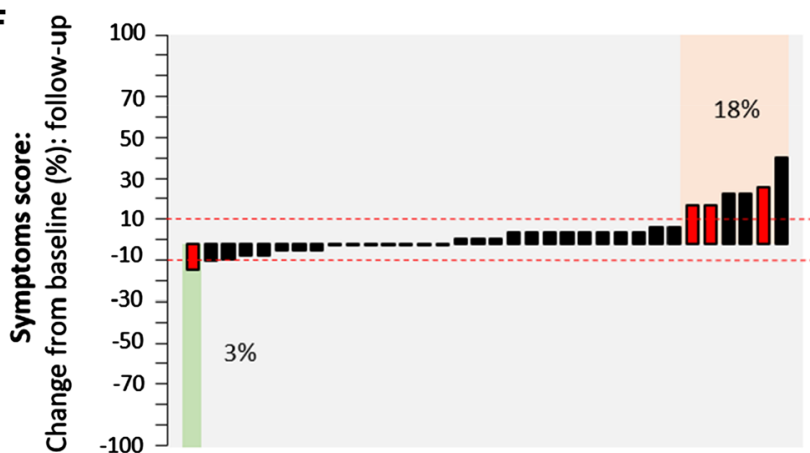

Fig. 2 Health-related quality of life according to EORTC-QLQ 30. Shows HRQOL score of 34 patients collected at baseline (before the first treatment) and at the first follow-up (4-15 weeks later) by analysing global health (a, b), function (c, d) and symptoms (e, f) score according to EORTC-QLQ30 version 3.0. For the global health and the function score, a high score indicates high health and for the symptom scale a low score indicates few symptoms. For general comparisons between baseline and the first follow-up,

By collecting data from different sites and countries across Europe, we could already show notable preliminary data regarding safety, bead size and periprocedural medications. In the final analysis, CIREL will elaborate on heterogeneity and homogeneity of compliance to guidelines and suggestions regarding technical aspects of treatment administration. boxplots were used (a, c, e). The difference between baseline and first follow-up was plotted for individual patients using a waterfall diagram. Cut-offs (dashed line) for clinically significant improvement were set at +10 for global health, functional score and -10 for symptom score and at -10 for global health, functional score and +10 for symptom score for deterioration. Red bars indicate patients with treatment intention: salvage therapy (see supplementary Table 1)

\section{Study Limitation}

The data for this interim analysis are from 50 patients from 9 centres in 7 European countries. The final data set will include patients from 25 different centres in 12 different countries. Therefore, conclusions could be subject to change as standard practices in different centres and countries could differ. Additionally, as analysing all effectiveness outcomes is beyond the scope of this interim analysis considering that follow-up data collection is 
ongoing and the results cannot yet be analysed in conjunction with outcomes such as overall survival, hepaticfree survival.

\section{Conclusion}

This interim analysis illustrates that in CIREL, LPirinotecan TACE was mainly used as salvage or intensification therapy with an acceptable toxicity profile. HRQOL of the global health score deteriorated in more patients than the functional and symptom score, for both of which over $70 \%$ of patients reported stable or improved scores at the first follow-up compared to baseline. Additionally, this interim analysis illustrates the feasibility of categorising the use of LP-irinotecan TACE and studying quality of life in terms of data completeness and distribution. Therefore, the final results of CIREL will be able to provide prospective and meaningful data for the use and safety of TACE using irinotecan in different settings across multiple sites in Europe and analyse HRQOL using the comprehensive EORTC questionnaire.

Acknowledgements We would like to thank Terumo Europe NV for providing an unrestricted educational grant that funded the study, Carole Déan for support with navigating the regulatory environment in France, the CIREL Steering Committee members Prof. Patrick Chevallier, Dr. Raul Garcia, Dr. Hassan Malik and Dr. Simon Pernot for their contribution to CIREL, as well as all participating centres and personnel involved in CIREL.

Funding This study was funded by an unrestricted educational grant provided by Terumo Europe NV.

\section{Compliance with Ethical Standards}

Conflict of interest Pereira reports the receipt of grants from Siemens Healthineers, Terumo, BTG and Biocompatibles, the receipt of honoraria from Terumo, Sirtex, Angiodynamics, Medtronic, Pharmacept and Guerbet, as well as study grants from BMS, MSD, GSK and Terumo and travel support from Bayer. B. Gonçalves reports personal fees from Medtronic, personal fees from Terumo, personal fees from Sirtex, outside the submitted work. T. Helmberger received speaker honoraria from SIRTEX Medical Europe. O. Pellerin has received personal fees from Merit Medical and shareholdings of COGITh-SAS. D. Arnold received consulting fees and speaker honoraria from TERUMO, Boston Scientific, SIRTEX Medical Europe and Biocompatibles. G. Maleux received speaker fees from SIRTEX Medical Europe. B. Sangro has received personal fees from Terumo and BTG, as well as personal fees and a grant from Sirtex Medical. J. Taieb reports receiving honoraria from Merck, Roche, Amgen, Lilly, Sanofi, Samsung, MSD, Servier, Celgene, Pierre Fabre; consulting or advisory Role for Roche, Merck KGaA, Amgen, Lilly, MSD, Servier, Pierre Fabre, Sanofi, Samsung; speakers' Bureau for Servier, Amgen, Roche, Sanofi, Merck, Lilly, Pierre Fabre. H. Prenen received honoraria and/or travel grants from Roche, Bayer, Amgen, Ipsen, Pfizer, Sanofi, Merck, Terumo and Lilly. R. Iezzi, R. Manfredi, F. Carchesio, Z. Bánsághi, E. Brountzos, S. Spiliopoulos, J. Echevarria-Uraga, R. Inchingolo, M. Nardella, M. Sousa, F.
T. Debaere, Gomez, B. Zeka, and N. Kaufmann report no conflict of interest.

Ethical Approval All procedures performed in studies involving human participants were in accordance with the ethical standards of the institutional and/or national research committee and with the 1964 Helsinki Declaration and its later amendments or comparable ethical standards.

Informed Consent Informed consent was obtained from all individual participants included in the study.

Consent to Publish For this type of study consent for publication is not required. However, consent for publication was obtained for every individual person's data included in the study.

Open Access This article is licensed under a Creative Commons Attribution 4.0 International License, which permits use, sharing, adaptation, distribution and reproduction in any medium or format, as long as you give appropriate credit to the original author(s) and the source, provide a link to the Creative Commons licence, and indicate if changes were made. The images or other third party material in this article are included in the article's Creative Commons licence, unless indicated otherwise in a credit line to the material. If material is not included in the article's Creative Commons licence and your intended use is not permitted by statutory regulation or exceeds the permitted use, you will need to obtain permission directly from the copyright holder. To view a copy of this licence, visit http://creativecommons. org/licenses/by/4.0/.

\section{References}

1. Van Cutsem E, Cervantes A, Adam R, et al. ESMO consensus guidelines for the management of patients with metastatic colorectal cancer. Ann Oncol. 2016. https://doi.org/10.1093/annonc/ mdw235.

2. Gruber-Rouh T, Naguib NNN, Eichler K, et al. Transarterial chemoembolization of unresectable systemic chemotherapy-refractory liver metastases from colorectal cancer: long-term results over a 10-year period. Int J Cancer. 2014. https://doi.org/10.1002/ ijc. 28443.

3. Bower M, Metzger T, Robbins K, et al. Surgical downstaging and neo-adjuvant therapy in metastatic colorectal carcinoma with irinotecan drug-eluting beads: a multi-institutional study. Hpb. 2016. https://doi.org/10.1111/j.1477-2574.2009.00117.x.

4. Vogl TJ, Gruber T, Balzer JO, et al. Repeated transarterial chemoembolization in the treatment of liver metastases of colorectal cancer: prospective study. Radiology. 2009. https://doi. org/10.1148/radiol.2501080295.

5. Mizandari M, Paksashvili N, Kikodze N, et al. Long-term survival in a patient with low-level inflammatory markers and liver metastasis, converted resectable by TACE. Immunotherapy. 2017. https://doi.org/10.2217/imt-2017-0069.

6. Narayanan G, Barbery K, Suthar R, et al. Transarterial chemoembolization using DEBIRI for treatment of hepatic metastases from colorectal cancer. Anticancer Res. 2013;33(5):2077-83.

7. Fiorentini G, Aliberti C, Tilli M, et al. Intra-arterial infusion of irinotecan-loaded drug-eluting beads (DEBIRI) versus intravenous therapy (FOLFIRI) for hepatic metastases from colorectal cancer: final results of a phase III study. Anticancer Res. 2012;32:1387-95.

8. Jones RP, Dunne D, Sutton P, et al. Segmental and lobar administration of drug-eluting beads delivering irinotecan leads 
to tumour destruction: a case-control series. Hpb. 2013. https:// doi.org/10.1111/j.1477-2574.2012.00587.x.

9. Boysen AK, Jensen M, Nielsen DT, et al. Cell-free DNA and chemoembolization in patients with liver metastases from colorectal cancer. Oncol Lett. 2018. https://doi.org/10.3892/ol.2018. 8925.

10. Martin RCG, Scoggins CR, Schreeder M, et al. Randomized controlled trial of irinotecan drug-eluting beads with simultaneous FOLFOX and bevacizumab for patients with unresectable colorectal liver-limited metastasis. Cancer. 2015. https:// doi.org/10.1002/cncr.29534.

11. Martin RCG, Robbins K, Tomalty D, et al. Transarterial chemoembolisation (TACE) using irinotecan-loaded beads for the treatment of unresectable metastases to the liver in patients with colorectal cancer: an interim report. World J Surg Oncol. 2009. https://doi.org/10.1186/1477-7819-7-80.

12. Martin RCG, Joshi J, Robbins K, et al. Transarterial chemoembolization of metastatic colorectal carcinoma with drug-eluting beads, irinotecan (DEBIRI): multi-institutional registry. J Oncol. 2009. https://doi.org/10.1155/2009/539795.

13. Pernot $\mathrm{S}$, Pellerin $\mathrm{O}$, Artru $\mathrm{P}$, et al. Intra-arterial hepatic beads loaded with irinotecan (DEBIRI) with mFOLFOX6 in unresectable liver metastases from colorectal cancer: a Phase 2 study. Br J Cancer. 2020. https://doi.org/10.1038/s41416-020-0917-4.

14. Pereira PL, Arnold D, De Baere T, et al. A multicentre, international, observational study on transarterial chemoembolisation in colorectal cancer liver metastases: design and rationale of CIREL. Dig Liver Dis. 2020. https://doi.org/10.1016/j.dld.2020. 05.051.

15. National Institutes of Health, National Cancer Institute. 1. CTCAE 4.03 Common Terminology Criteria for Adverse Events (CTCAE) Version 4.0. May 28, 2009 (v4.03: June 14, 2010). U.S. Department of Health and Human Services. https://evs.nci.nih. gov/ftp1/CTCAE/CTCAE_4.03/CTCAE_4.03_2010-06-14_ QuickReference_5x7.pdf. Accessed 11 Aug 2020.

16. Common Terminology Criteria for Adverse Events (CTCAE) Version 5.0. November 27, 2017. U.S. Department of Health and Human Services. https://ctep.cancer.gov/protocoldevelopment/ electronic_applications/docs/CTCAE_v5_Quick_Reference_5x7. pdf. Accessed 11 Aug 2020.

17. Flechtner H, Bottomley A. Quality of life assessment and research in the EORTC (European Organisation for Research and Treatment of Cancer). Oncologie. 2006;8:443-6. https://doi.org/ 10.1007/s10269-006-0412-4.

18. Aaronson NK, Ahmedzai S, Bergman B, Bullinger M, Cull A, Duez NJ, Filiberti A, Flechtner H, Fleishman SB, de Haes JCJM, Kaasa S, Klee MC, Osoba D, Razavi D, Rofe PB, Schraub S, Sneeuw KCA, Sullivan M, Takeda F. The European Organisation for Research and Treatment of Cancer QLQ-C30: a quality-of-life instrument for use in international clinical trials in oncology. J Natl Cancer Inst. 1993;85:365-76.

19. Fayers PM, Aaronson NK, Bjordal K, Groenvold M, Curran D, Bottomley A, on behalf of the EORTC Quality of Life Group. The EORTC QLQ-C30 Scoring Manual (3rdEdition). Published by: European Organisation for Research and Treatment of Cancer, Brussels 2001.

20. Cocks K, King MT, Velikova G, et al. Evidence-based guidelines for interpreting change scores for the European Organisation for the Research and Treatment of Cancer Quality of Life Questionnaire Core 30. Eur J Cancer. 2012. https://doi.org/10.1016/j. ejca.2012.02.059.

21. Vogel A, Cervantes A, Chau I, et al. Hepatocellular carcinoma: ESMO clinical practice guidelines for diagnosis, treatment and follow-up. Ann Oncol. 2018;29:iv238-iv255. https://doi.org/10. 1093/annonc/mdy308.
22. ESMO Guidelines Committee. eUpdate Hepatocellular Carcinoma Treatment Recommendations. 2020. https://www.esmo. org/guidelines/gastrointestinal-cancers/hepatocellular-carcinoma/ eupdate-hepatocellular-carcinoma-treatment-recommendations3. Accessed 13 Aug 2020.

23. Lévi FA, Boige V, Hebbar M, et al. Conversion to resection of liver metastases from colorectal cancer with hepatic artery infusion of combined chemotherapy and systemic cetuximab in multicenter trial OPTILIV. Ann Oncol. 2016. https://doi.org/10. 1093/annonc/mdv548.

24. Martinelli E, Ciardiello D, Martini G, et al. Implementing antiepidermal growth factor receptor (EGFR) therapy in metastatic colorectal cancer: challenges and future perspectives. Ann Oncol. 2020. https://doi.org/10.1016/j.annonc.2019.10.007.

25. Kim SY, Kim TW. Current challenges in the implementation of precision oncology for the management of metastatic colorectal cancer. ESMO Open. 2020. https://doi.org/10.1136/esmoopen2019-000634.

26. Kreidieh M, Mukherji D, Temraz S, et al. Expanding the scope of immunotherapy in colorectal cancer: current clinical approaches and future directions. Biomed Res Int. 2020. https://doi.org/10. 1155/2020/9037217.

27. Iezzi R, Marsico VA, Guerra A, et al. Trans-arterial chemoembolization with irinotecan-loaded drug-eluting beads (DEBIRI) and capecitabine in refractory liver prevalent colorectal metastases: a phase II single-center study. Cardiovasc Intervent Radiol. 2015. https://doi.org/10.1007/s00270-015-1080-9.

28. Ngo A, von Stempel C, Corbo B, et al. Transarterial chemoembolisation of colorectal liver metastases with irinotecan-loaded beads: a bi-institutional analysis of 125 treatments in 53 [atients. Cardiovasc Interv Radiol. 2019. https://doi.org/10.1007/s00270019-02219-4.

29. Mauri G, Varano GM, Della Vigna P, et al. Transarterial embolization with small-size particles loaded with irinotecan for the treatment of colorectal liver metastases: results of the MIRACLE III Study. Cardiovasc Interv Radiol. 2018. https://doi. org/10.1007/s00270-018-2017-x.

30. Ranieri G, Asabella AN, Altini C, et al. A pilot study employing hepatic intra-arterial irinotecan injection of drug-eluting beads as salvage therapy in liver metastatic colorectal cancer patients without extrahepatic involvement: the first southern Italy experience. Onco Targets Ther. 2016. https://doi.org/10.2147/OTT. S112670.

31. Bhutiani N, Akinwande O, Martin RCG. Efficacy and toxicity of hepatic intra-arterial drug-eluting (Irinotecan) bead (DEBIRI) therapy in irinotecan-refractory unresectable colorectal liver metastases. World J Surg. 2016. https://doi.org/10.1007/s00268015-3386-9.

32. Lencioni R, Aliberti C, De Baere T, et al. Transarterial treatment of colorectal cancer liver metastases with irinotecan-loaded drugeluting beads: technical recommendations. J Vasc Interv Radiol. 2014. https://doi.org/10.1016/j.jvir.2013.11.027.

33. Iezzi R, Kovacs A, Prenen H, et al. Transarterial chemoembolisation of colorectal liver metastases with irinotecan-loaded beads: what every interventional radiologist should know. Eur J Radiol Open. 2020. https://doi.org/10.1016/j.ejro.2020.100236.

34. Dreher MR, Sharma KV, Woods DL, et al. Radiopaque drugeluting beads for transcatheter embolotherapy: experimental study of drug penetration and coverage in swine. J Vasc Interv Radiol. 2012. https://doi.org/10.1016/j.jvir.2011.10.019.

35. Lewis AL, Dreher MR, O'Byrne V, et al. DC BeadM1TM: towards an optimal transcatheter hepatic tumour therapy. J Mater Sci Mater Med. 2016. https://doi.org/10.1007/s10856-015-56296.

36. Akinwande O, Scoggins C, Martin RCG. Early experience with 70-150 $\mathrm{m}$ irinotecan drug-eluting beads (M1-DEBIRI) for the 
treatment of unresectable hepatic colorectal metastases. Anticancer Res. 2016;36:3413-8.

37. Fereydooni A, Letzen B, Ghani MA, et al. Irinotecan-eluting 75-150- $\mu \mathrm{m}$ embolics lobar chemoembolization in patients with colorectal cancer liver metastases: a prospective single-center phase I study. J Vasc Interv Radiol. 2018. https://doi.org/10.1016/ j.jvir.2018.08.010.

38. Young S, D'Souza D, Flanagan S, Golzarian J. Review of the clinical evidence for the use of DEBIRI in the treatment of colorectal metastatic disease. Cardiovasc Interv Radiol. 2017. https://doi.org/10.1007/s00270-016-1537-5.

39. Boeken T, Moussa N, Pernot S, et al. Does bead size affect patient outcome in irinotecan-loaded beads chemoembolization plus systemic chemotherapy regimens for liver-dominant colorectal cancer? Results of an observational study. Cardiovasc Interv Radiol. 2020. https://doi.org/10.1007/s00270-020-02438-0.

40. Fiorentini G, Sarti D, Nani R, et al. Updates of colorectal cancer liver metastases therapy: review on DEBIRI. Hepatic Oncol. 2020. https://doi.org/10.2217/hep-2019-0010.

41. Eichler K, Zangos S, Mack MG, et al. First human study in treatment of unresectable liver metastases from colorectal cancer with irinotecan-loaded beads (DEBIRI). Int J Oncol. 2012. https://doi.org/10.3892/ijo.2012.1572.

42. Scevola G, Loreni G, Rastelli M, et al. Third-line treatment of colorectal liver metastases using DEBIRI chemoembolization. Med Oncol. 2017. https://doi.org/10.1007/s12032-017-0890-9.
43. Martin RCG, Joshi J, Robbins K, et al. Hepatic intra-arterial injection of drug-eluting bead, irinotecan (DEBIRI) in unresectable colorectal liver metastases refractory to systemic chemotherapy: results of multi-institutional study. Ann Surg Oncol. 2011. https://doi.org/10.1245/s10434-010-1288-5.

44. Aliberti C, Fiorentini G, Muzzio PC, et al. Trans-arterial chemoembolization of metastatic colorectal carcinoma to the liver adopting DC Bead ${ }^{\circledR}$, drug-eluting bead loaded with irinotecan: results of a phase II clinical study. Anticancer Res. 2011:31:4581-7.

45. Fiorentini G, Carandina R, Sarti D, et al. Polyethylene glycol microspheres loaded with irinotecan for arterially directed embolic therapy of metastatic liver cancer. World J Gastrointest Oncol. 2017. https://doi.org/10.4251/wjgo.v9.i9.379.

46. Fiorentini G, Aliberti C, Turrisi G, et al. Intraarterial hepatic chemoembolization of liver metastases from colorectal cancer adopting irinotecan-eluting beads: results of a phase II clinical study. Vivo (Brooklyn). 2007;21:1085-92.

47. Fiorentini G, Sarti D, Aliberti C, et al. Chemoembolization in conjunction with bevacizumab: preliminary results. J Vasc Interv Radiol. 2018. https://doi.org/10.1016/j.jvir.2018.04.022.

Publisher's Note Springer Nature remains neutral with regard to jurisdictional claims in published maps and institutional affiliations. 